\title{
Long-term relapses after 12-month treatment for Mycobacterium kansasii
} lung disease

\author{
M. Santin*, J. Dorca*, F. Alcaide『, L. Gonzalez*, S. Casas*, \\ M. Lopez ${ }^{\#}$ and M.R. Guerra*
}

ABSTRACT: Long-lasting therapy for Mycobacterium kansasii lung disease with rifampincontaining multidrug regimens is needed to avoid relapses. The aim of the present study is to evaluate a short multidrug treatment regimen for $M$. kansasii lung disease.

A retrospective observational study of 75 patients with $M$. kansasii lung disease was conducted in a teaching hospital from January 1990 to December 2005.

In total, 75 (67.6\%) out of 111 patients diagnosed with $M$. kansasii lung disease completed a 12month multidrug treatment regimen, including rifampin, isoniazid and ethambutol, supplemented with streptomycin during the first 2-3 months. After a 41.5-month median follow-up, five (6.6\%) patients relapsed. The relapse rate was 2.19 (95\% confidence interval $0.71-5.12)$ per 100 person $\cdot y r s$. Treatment compliance was considered to be appropriate in all five patients and no drug resistance developed in any case.

In conclusion, a 12-month fixed-course treatment is effective in most cases of Mycobacterium kansasii lung disease, but may not be long enough for all patients.

\section{KEYWORDS: Mycobacterium kansasii, relapse, short treatment}

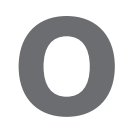
ne of the most pathogenic and frequent nontuberculous mycobacteria (NTM) isolated throughout the world is Mycobacterium kansasii [1-3]. Long-lasting chemotherapy with rifampin-containing multidrug regimens is needed to avoid relapses [4]. In the pre-rifampin period, only $52-80 \%$ of patients achieved sputum conversion at 6 months, relapses appeared in $\sim 10 \%$ and surgical resection was frequently required [5]. With rifampin-containing regimens, sputum conversion within the first 4 months of treatment is the rule, and treatment failures and long-term relapses occur in $\sim 1 \%$ of cases if treatment is maintained longer than 12 months [5-8]. Maintaining chemotherapy for at least 12 months after conversion of sputum, with a multidrug regimen, is currently recommended by the American Thoracic Society (ATS) and the Infectious Diseases Society of America [4], whereas a two-drug regimen of 9 months is recommended by the British Thoracic Society (BTS) as treatment for M. kansasii lung disease [9].

As controlled trials for NTM infections are often difficult to conduct, large retrospective observational studies may be helpful to evaluate alternative treatment strategies. Based on the results of a study of 12 months of treatment with an initial phase of intermittent streptomycin [10] and the current authors' own favourable experience with a similar regimen in HIV-infected patients, a 12-month course of chemotherapy with rifampin, isoniazid and ethambutol, with streptomycin for the first 3 months, was adopted as the standard treatment for disease caused by $M$. kansasii in the Bellvitge University Hospital (HUB; Barcelona, Spain) in 1990. The aim of the present study was to evaluate the efficacy of the 12-month treatment regimen for $M$. kansasii lung disease.

\section{MATERIAL AND METHODS \\ Study subjects}

Patients were identified by reviewing the register of positive cultures at the HUB from January 1, 1990 to December 31, 2005. M. kansasii lung disease was diagnosed according to the ATS criteria [4]. Data were retrieved from the individual registers available at the tuberculosis control unit (TCU) and from clinical charts of each patient. Patients with known HIV infection were excluded.

\section{Study design and setting}

The present study was a retrospective observational study performed at the TCU of the HUB, a 900-bed teaching hospital in the metropolitan area of Barcelona, which admits only adults.

\section{AFFILIATIONS}

Depts of *Infectious Diseases,

${ }^{\#}$ Respiratory Medicine and

"Microbiology, Mycobacteriology

Section, Bellvitge Institute for

Biomedical Research (IDIBELL),

Bellvitge University Hospital,

University of Barcelona,

Barcelona, Spain.

\section{CORRESPONDENCE}

M. Santin

Dept of Infectious Diseases Institut d'Investigació Biomèdica de Bellvitge (IDIBELL)

Hospital Universitari de Bellvitge

University of Barcelona

C/ Feixa Llarga s/n 08907

L'Hospitalet de Llobregat

Barcelona

Spain

Fax: 34932607637

E-mail: msantin@bellvitgehospital.cat

Received:

February 152008

Accepted after revision:

September 142008

\section{SUPPORT STATEMENT}

This study received financial support from the Spanish Ministry of Health Services and Consumer Affairs (Madrid, Spain), the Health Institute Carlos III (Madrid) and the Spanish Network for Research in Infectious Diseases (REIPI; RD06/0008).

\section{STATEMENT OF INTEREST}

None declared.

European Respiratory Journal Print ISSN 0903-1936 Online ISSN 1399-3003 
Approval was obtained from the ethics committee of the HUB (CEIC number 177/07). Patients diagnosed with either tuberculosis or NTM infection were referred to the TCU for treatment, unless they were HIV positive. The treatment schedule for M. kansasii lung disease consisted of oral rifampin $\left(600 \mathrm{mg} \cdot \mathrm{day}^{-1}\right)$, isoniazid $\left(300 \mathrm{mg} \cdot \mathrm{day}^{-1}\right)$ and ethambutol $\left(25 \mathrm{mg} \cdot \mathrm{kg}^{-1} \cdot\right.$ day $^{-1}$ during the first 2 months, $15 \mathrm{mg} \cdot \mathrm{kg}^{-1} \cdot \mathrm{day}^{-1}$ thereafter) for a 12-month period, with i.m. streptomycin (750$1,000 \mathrm{mg}$ two to three times per week) during the first 3 months. Patients were treated and followed-up according to an established protocol, which included regular nurse and medical visits for assessment of clinical and microbiological evolution, treatment compliance and adverse effects of drugs. Nonattendance at visits was registered and attempts were made to recover missing patients. Microbiological response was monitored by means of sputum smear and culture (whenever sputum could be obtained) every 2 months throughout the treatment period. Chest radiographs were taken at month 2 of treatment and then every 4-6 months if clinical and microbiological evolution was satisfactory. All patients underwent periodic ophthalmological examination, including visual acuity, colour vision and visual field. Posttreatment follow-up with sputum examination and chest radiograph was maintained for a minimum of 3 yrs. However, follow-up could be lengthened or even continued indefinitely due to the concomitant chronic pulmonary disease. Adherence to treatment was initially assessed by asking whether the prescribed treatment had been followed or not and by attendance at scheduled visits. Moreover, since the year 2000, isoniazid metabolites in urine and/or urine colour were tested at every visit [11]. Directly observed therapy was not regularly offered to patients with NTM infection. Specific information on the diagnosis, clinical picture, radiology, treatment, adherence and follow-up of the mycobacterial disease for each patient was entered into a database at the TCU.

\section{Microbiological studies}

Clinical specimens were processed following the conventional digestion-decontamination procedure [12]. Smears were stained by auramine-rhodamine fluorochrome. Positive slides were confirmed by the Ziehl-Neelsen stain [13]. Samples $(0.5 \mathrm{~mL})$ of specimens were inoculated into BACTEC $12 \mathrm{~B}$ vials and onto Löwenstein-Jensen medium slants. The liquid cultures were performed, from 1990 to 1998, using the BACTEC 460 system (Becton Dickinson, Sparks, MD, USA) and, from 1999 onwards, using the MB/BacT system (BioMérieux SA, Marcy l'Etoile, France). Isolates were identified by conventional biochemical and culture tests, PCRrestriction fragment length polymorphism analysis of the $h s p 65$ gene and DNA probes (AccuProbe; GenProbe Inc., San Diego, CA, USA). Susceptibility testing was performed with the radiometric (BACTEC) susceptibility test (Becton Dickinson), based on the interpretative criteria used with Mycobacterium tuberculosis [14-16]. Susceptibility breakpoints adopted were as follows: $1 \mu \mathrm{g} \cdot \mathrm{mL}^{-1}$ for isoniazid and rifampin, $5 \mu \mathrm{g} \cdot \mathrm{mL}^{-1}$ for ethambutol, and $2 \mu \mathrm{g} \cdot \mathrm{mL}^{-1}$ for streptomycin.

\section{Definitions}

Patients were considered to be cured if, at the end of the treatment, they presented resolution of symptoms, disappearance or improvement of radiological alterations and sputum negativity, when available. If sputum could not be obtained at the end of treatment, in addition to the clinical and radiological improvement, at least one negative sputum culture during treatment was mandatory to consider a patient as cured. Relapse was defined as the occurrence, after conversion to negative and completion of a full course of treatment, of two or more positive sputum cultures during a period of $>2$ weeks, regardless of radiographic appearance. Alternatively, the occurrence of two or more positive sputum cultures with positive smear $\geqslant 1$ day apart and radiographic changes was also accepted. Treatment was considered to begin on the date when at least rifampin and ethambutol were started. Compliance was considered appropriate whenever attendance at visits was regular and the urine examination (colour and/or isoniazid metabolites) was positive in $>80 \%$ of visits.

\section{Data analysis}

The main end-point of evaluation was the proportion of patients who relapsed. Relapses were expressed as cumulative incidence and rates per person-yr, to adjust for differences in follow-up (incidence density). Since the chance of relapse did not remain stable indefinitely (most relapses occurring in the first years after stopping treatment), all cases were censored after completing the fifth year of follow-up.

\section{RESULTS}

\section{Baseline characteristics and treatment regimens}

Of the 111 patients with $M$. kansasii lung disease, $92(82.8 \%)$ completed a full course of treatment. Of these, $79(85.9 \%)$ patients were treated for 12 months with a combination of rifampin, isoniazid and ethambutol. In 66 (83.5\%) of these patients, this was supplemented with streptomycin for the first 3 months. In the remaining 13 patients, streptomycin was skipped because of toxicity concerns $(n=5)$, known allergy $(n=1)$ or other reasons $(n=7)$. Four patients were not included in the analysis: one of them was retreated because of positive culture (however, relapse criteria were not fulfilled); and the other three had no follow-up to be assessed. In total, 75 patients were finally included for the analysis. Of these, 37 $(49.3 \%)$ patients were HIV negative, while in the other 38 $(50.7 \%)$, HIV status was unknown. No significant difference was found between patients who underwent HIV testing and those who did not, except that chronic pulmonary disease was more frequent in those who did not undergo testing, and there was a lower mean age in those who were tested $(76.0 \%$ versus $44.4 \%(p=0.04)$ and 53.0 versus 42.6 yrs $(p=0.002)$ for those not tested versus those who underwent testing, respectively). The main baseline characteristics of the 75 patients are shown in table 1 . Most patients were males in the sixth decade of life with some comorbidity, mainly chronic pulmonary disease. Upper-lobe cavitated infiltrates were the predominant radiological finding. Sputum smear was positive in $>65 \%$ of cases. Strain sensitivity was available in $48(64.0 \%)$ cases. Isolates from $38(79.2 \%)$ of these patients were susceptible to all the drugs. Of the nine strains with some degree of resistance, eight had a minimum inhibitory concentration (MIC) of $5 \mu \mathrm{g} \cdot \mathrm{mL}^{-1}$ for isoniazid and one had an $\mathrm{MIC}$ of $2 \mu \mathrm{g} \cdot \mathrm{mL}^{-1}$ for rifampin. 


\begin{tabular}{|c|c|c|}
\hline TABLE 1 & \multicolumn{2}{|c|}{$\begin{array}{l}\text { Baseline characteristics, follow-up and relapses } \\
\text { of } 75 \text { patients with Mycobacterium kansasii } \\
\text { lung disease }\end{array}$} \\
\hline Age yrs & & $49.5 \pm 13.3$ \\
\hline Sex male & & $71(94.7)$ \\
\hline \multicolumn{3}{|c|}{ Comorbid conditions } \\
\hline Smoking & & $65(86.7)$ \\
\hline $\mathrm{COPD}^{\#}$ & & $33(44.0)$ \\
\hline Previous tu & erculosis & $17(23.6)^{\S}$ \\
\hline Diabetes $\mathrm{m}$ & ellitus & $8(10.7)$ \\
\hline Malignancy & & $7(9.3)$ \\
\hline Alcohol ab & & $24(32.0)$ \\
\hline None & & $24(32.4)^{f}$ \\
\hline Cavitation o & chest radiograph & $54(72.0)$ \\
\hline Positive sme & & $49(66.2)^{f}$ \\
\hline \multicolumn{3}{|c|}{ Duration of treatment months } \\
\hline Rifampin & & $12.5 \pm 0.7$ \\
\hline Isoniazid & & $12.2 \pm 1.6$ \\
\hline Ethambuto & & $11.9 \pm 1.2$ \\
\hline Streptomyc & & $2.2 \pm 0.5$ \\
\hline Good treatm & ent compliance & $69(92.0)$ \\
\hline Duration of & llow-up after treatment months & $50.1 \pm 36.1$ \\
\hline \multicolumn{3}{|c|}{ Relapses $^{+}$} \\
\hline Cumulative & ncidence rate & $5(6.6)$ \\
\hline Incidence r & te per 100 patient-yrs & $2.19(0.71-5.12)$ \\
\hline \multicolumn{3}{|c|}{$\begin{array}{l}\text { Data are presented as mean } \pm \mathrm{SD}, \mathrm{n}(\%) \text { or rate }(95 \% \text { confidence interval). } \\
\text { COPD: chronic obstructive pulmonary disease. }{ }^{*}: \text { includes COPD and other } \\
\text { chronic pulmonary diseases such as pneumoconiosis and bronchiectasis; } \\
\text { for sputum or bronchial washing; }{ }^{+}: \text {calculated for patients who had at least one } \\
\text { visit after stopping treatment, and accounting for no more than } 5 \text { yrs of follow- } \\
\text { up; }^{\text {s. }} \text { : data for } 72 \text { patients; }{ }^{f} \text { : data for } 74 \text { patients. }\end{array}$} \\
\hline
\end{tabular}

\section{Outcome}

During follow-up, five (6.6\%) out of 75 patients relapsed after a median (range) of 41.5 (11-48) months. The incidence density rate was 2.19 (95\% confidence interval 0.71-5.12) per 100 patient-yrs (table 1). All five relapses occurred among patients treated with the combination that included streptomycin. There were no relevant differences regarding main characteristics between patients who relapsed and those who did not, apart from a higher proportion of patients with positive sputum smear in the nonrelapse group and a trend towards higher comorbidity in the relapse group (0 versus $65.7 \%$ positive sputum smear $(p=0.007)$ and 100 versus $70.0 \%$ with comorbities $(\mathrm{p}=0.18)$ for relapsed versus nonrelapsed, respectively; table 2). Although not statistically significant, there was a trend towards a relationship between age and relapse: none of the 17 patients $\leqslant 40$ yrs of age relapsed, compared with five out of the 58 patients $>40$ yrs of age $(\mathrm{p}=0.27)$. Treatment compliance was considered to be appropriate in the five relapse patients, and the susceptibility test, which was available for all five cases, showed susceptibility to the four drugs tested (rifampin, isoniazid, ethambutol and streptomycin) before the treatment and at the time of relapse. In two out of the five patients, streptomycin was discontinued after 2 and 4 weeks, respectively, because of dizziness. Table 3 provides detailed information on the five relapses. The five patients were retreated with the same drugs

\begin{tabular}{|c|c|c|c|c|}
\hline \multirow{2}{*}{ TABLE 2} & \multicolumn{4}{|c|}{$\begin{array}{l}\text { Risk factors for relapsing in } 75 \text { patients with } \\
\text { Mycobacterium kansasii lung disease }\end{array}$} \\
\hline & & Relapse & Nonrelapse & p-value \\
\hline \multicolumn{2}{|l|}{ Patients n } & 5 & 70 & \\
\hline \multicolumn{2}{|l|}{ Age yrs } & $55 \pm 4.9$ & $48.9 \pm 1.6$ & 0.33 \\
\hline \multicolumn{2}{|l|}{ Sex male } & $4(80.0)$ & $64(91.4)$ & 0.40 \\
\hline \multicolumn{5}{|c|}{ Comorbidities $^{\#}$} \\
\hline \multicolumn{2}{|l|}{ Any } & $5(100)$ & $49(70.0)$ & 0.18 \\
\hline \multicolumn{2}{|l|}{0} & 0 & $21(30.0)$ & 0.18 \\
\hline \multicolumn{2}{|l|}{1} & $1(20.0)$ & $18(25.7)$ & 0.63 \\
\hline \multicolumn{2}{|l|}{$>1$} & $4(80.0)$ & $31(44.3)$ & 0.14 \\
\hline \multicolumn{5}{|c|}{$\begin{array}{l}\text { Duration of treatment months } \\
\text { mean (IQR) }\end{array}$} \\
\hline \multicolumn{2}{|c|}{ Rifampin } & $12.0(1.0)$ & $12.5(1.0)$ & 0.76 \\
\hline \multicolumn{2}{|l|}{ Isoniazid } & $12.0(1.0)$ & $12.5(1.0)$ & 0.94 \\
\hline \multicolumn{2}{|l|}{ Ethambutol } & $12.0(0.0)$ & $11.9(0.0)$ & 0.64 \\
\hline \multicolumn{2}{|l|}{ Streptomycin } & $2.0(1.8)$ & $2.0(1.0)$ & 0.10 \\
\hline \multicolumn{5}{|c|}{ Chest radiograph } \\
\hline \multicolumn{2}{|c|}{ Cavitation } & $3(60.0)$ & $49(70.0)$ & 0.49 \\
\hline \multicolumn{2}{|c|}{ Bilateral involvement } & $2(40.0)$ & $17(24.3)$ & 0.37 \\
\hline \multicolumn{2}{|c|}{ Positive sputum smear } & 0 & $46(65.7)$ & 0.007 \\
\hline \multicolumn{2}{|c|}{ Good treatment compliance } & $5(100)$ & $64(91.4)$ & 0.51 \\
\hline \multicolumn{5}{|c|}{$\begin{array}{l}\text { Data are presented as } n(\%) \text { or mean } \pm S D \text {, unless otherwise stated. IQR: } \\
\text { interquartile range. }{ }^{\#}: \text { including chronic obstructive pulmonary disease, alcohol } \\
\text { abuse, diabetes mellitus, chronic liver disease, immunosuppressive therapy, } \\
\text { malignancy, gastrectomy and chronic pancreatitis. }\end{array}$} \\
\hline
\end{tabular}

used in the first episode, for 18 months. After a median (range) of follow-up of 5 (3.5-8) yrs, they did not have further relapses.

Regarding safety, 11 (14.7\%) of the 75 patients suffered some kind of adverse event, which resulted in discontinuation of some drug in seven $(9.3 \%)$ cases. Dizziness, presumably caused by the streptomycin, was the most frequent cause of treatment discontinuation (four $(6.3 \%)$ out of the 63 treated with streptomycin). Isoniazid was discontinued in three $(4.0 \%)$ cases, ethambutol in two $(2.7 \%)$, and all treatment drugs in one $(1.3 \%)$ case.

In total, $28(25.2 \%)$ of the 111 initial patients died, 13 of them before finishing treatment. Death was not related to $M$. kansasii disease or to treatment in any of these cases. Malignancy, mainly lung and throat neoplasm, was the cause of death in 14 $(50 \%)$ cases.

\section{DISCUSSION}

The results of the present study indicate that a 12-month, fixedcourse treatment with the currently used drugs, although effective in most cases, does not cure all patients with M. kansasii lung disease. The observed relapse rate of $6.6 \%$ (2.19 per 100 person-yrs) is higher than that reported in previous studies with longer rifampin-containing regimens $[5,6,8]$.

Several studies have examined shorter regimens for $M$. kansasii lung disease [10, 17-19]. In a large retrospective study from the Czech Republic, 417 patients were treated with various multidrug regimens for 9-12 months and 8\% relapsed after 


\section{TABLE 3 Main characteristics of five relapses}

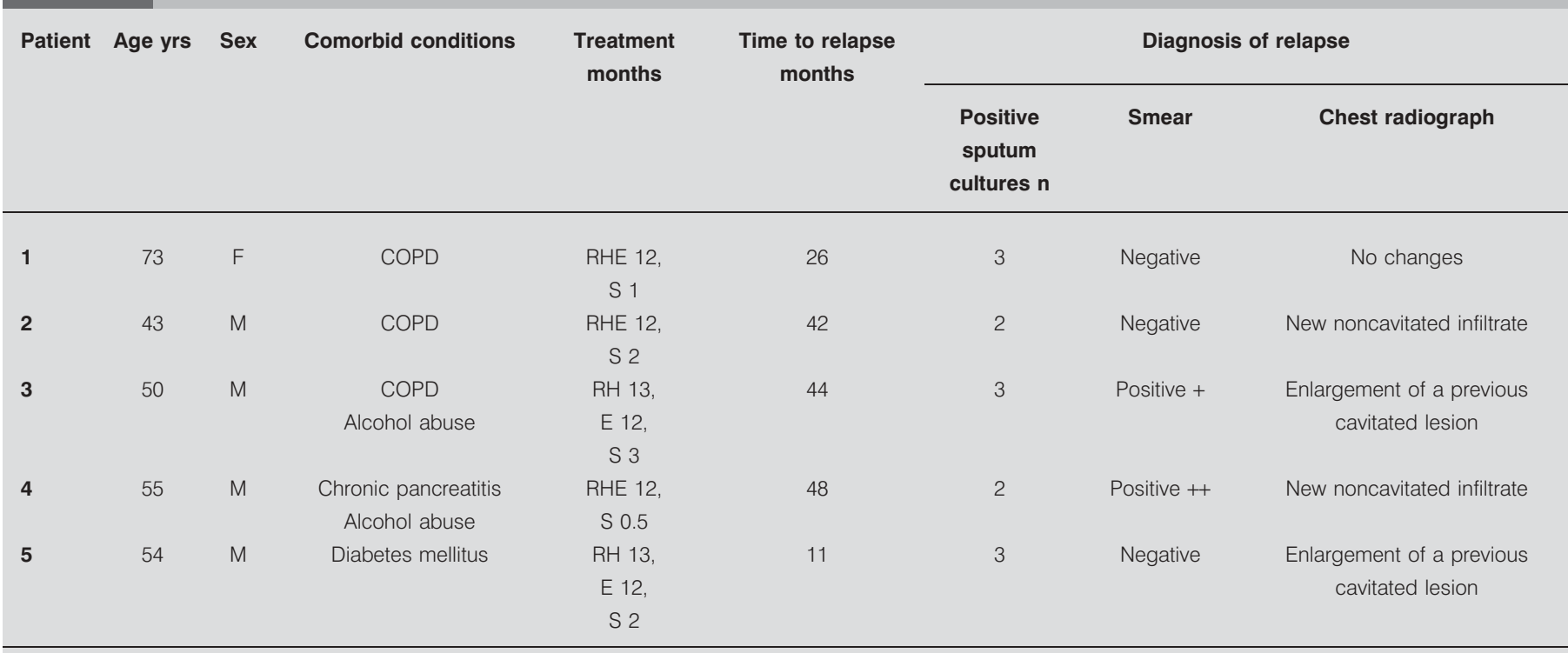

F: female; M: male; COPD: chronic obstructive pulmonary disease; R: rifampin; H: isoniazid; E: ethambutol; S: streptomycin.

1-7 yrs' follow-up [17]. A small prospective study compared 12-month and 18-month regimens [18]. In that study, 28 patients received rifampin, isoniazid and ethambutol daily for 6 months, followed by rifampin and isoniazid to complete either 12 or 18 months of therapy. One $(7 \%)$ patient out of the 14 treated for 12 months relapsed after 12-30 months of follow-up. In a prospective study of a 9-month treatment schedule with rifampin and ethambutol conducted by the BTS, $10 \%$ of patients relapsed within $5 \mathrm{yrs}$ of stopping treatment [19]. AHN et al. [10] conducted a prospective study in which 40 patients were treated with $1 \mathrm{~g}$ of streptomycin twice weekly for the first 3 months, added to the three-drug background scheme consisting of a rifampin, isoniazid and ethambutol regimen for 12 months. Only one (2.5\%) patient relapsed 6 months after completing treatment. However, only 12 out of the 40 patients were followed up for as long as 4 yrs, which might explain the low relapse rate observed. Indeed, in the present study, relapses occurred after a mean of 42 months after completing treatment, and three (out of five) patients relapsed after $>3$ yrs of follow-up, which means that relapse rates may be underestimated if followup is too short.

The current authors were unable to find any factors that could identify those patients prone to relapse. Demographic, clinical and radiographic characteristics of the five patients who relapsed were comparable to those of the ones who did not. The finding that relapses occurred in patients with negative sputum smear had not been reported previously, and it does not have a convincing explanation. In one case, relapse seemed likely to be related to the slow microbiological response, since sputum culture became negative only after 6 months of treatment and lung cavitations persisted on the chest radiograph at the end of treatment. The remaining patients presented certain characteristics that may potentially contribute to relapse. Although not statistically significant, there was a trend towards an association of relapse with older age and presence of comorbidities. In fact, no relapses occurred in patients $<40$ yrs of age and without debilitating conditions.

Whether relapses could be completely prevented with longer treatment regimens is not known. Some cases considered as relapses may actually be exogenous re-infections, as has been strongly suggested in some reports $[8,19]$. In fact, $M$. kansasii is a ubiquitous pathogen to which people may be repeatedly exposed and become infected more than once. It may, therefore, not be possible to avoid new episodes of active disease completely even with treatments of longer duration. However, in the present series, the five patients who relapsed were retreated with the same drugs for 18 months, and after a median of 5 yrs after finishing treatment none relapsed again, which supports the idea that some patients require longer courses of treatment.

While the current results do not allow conclusions to be drawn on the optimal length of chemotherapy for lung disease caused by M. kansasii, they did not support the use of a fixed short course of treatment in all cases. A 12-month regimen, as long as it includes rifampin and ethambutol, is probably appropriate in younger patients without debilitating conditions. For the rest, it appears advisable to treat for longer periods of time, guided by periodic sputum cultures. The ATS's current recommendation [4] of maintaining chemotherapy for a minimum of 12 months' negative sputum cultures seems to be judicious. Future research into M. kansasii should address the development of new treatment regimens with new active drugs. Clarithromycin and newer fluoroquinolones have excellent in vitro activity against M. kansasii, but clinical experience with these drugs is negligible [20-23]. Recently, a favourable experience with an intermittent regimen of clarithromycin, ethambutol and rifampin has been reported [23]. 
The main shortcoming of the present study is its retrospective design. Since it is not a controlled trial, treatment given in each case might have been influenced by considerations of the patients' clinical conditions, toxicity concerns and likelihood of adherence to treatment. In fact, although a 12-month duration has been adopted as the standard of care, 20 patients were treated either for 18 months or without streptomycin. HIV determination was available in $<50 \%$ of patients. However, misclassification of HIV-seropositive patients as HIV seronegative in the current series is unlikely. First, in the TCU of the HUB, HIV tests are performed routinely in patients with risk factors. Secondly, whereas $M$. kansasii disease among HIV patients mostly appears with advanced AIDS, it is disseminated in a significant number of cases, and death ensues if effective antiretroviral therapy is not given. In the present series, no patients had disseminated disease and none developed AIDS-associated complications or died of AIDS after extended follow-up.

In summary, although a 12-month fixed-course treatment is effective in most cases of Mycobacterium kansasii disease, it does not cure all patients. While the results of the newest active drugs are awaited, the current practice of maintaining chemotherapy for at least 12 months after sputum culture becomes negative appears sensible.

\section{REFERENCES}

1 Wolinsky E. Nontuberculous mycobacteria and associated diseases. Am Rev Respir Dis 1979; 119: 107-159.

2 Bittner MJ, Horowitz EA, Safranek TJ, Preheim LC. Emergence of Mycobacterium kansasii as the leading mycobacterial pathogen isolated over a 20-year period at a midwestern Veterans Affairs hospital. Clin Infect Dis 1996; 22: 1109-1110.

3 Bloch KC, Zwerling L, Pletcher MJ, et al. Incidence and clinical implications of isolation of Mycobacterium kansasii: results of a 5-year, population-based study. Ann Intern Med 1998; 129: 698-704.

4 Griffith DE, Aksamit T, Brown-Elliott BA, et al. An official ATS/IDSA statement: diagnosis, treatment, and prevention of nontuberculous mycobacterial diseases. Am J Respir Crit Care Med 2007; 175: 367-416.

5 Pezzia W, Raleigh JW, Bailey MC, Toth EA, Silverblatt J. Treatment of pulmonary disease due to Mycobacterium kansasii: recent experience with rifampin. Rev Infect Dis 1981; 3: 1035-1039.

6 Ahn CH, Lowell JR, Ahn SS, Ahn S, Hurst GA. Chemotherapy for pulmonary disease due to Mycobacterium kansasii: efficacies of some individual drugs. Rev Infect Dis 1981; 3: 1028-1034.

7 Banks J, Hunter AM, Campbell IA, Jenkins PA, Smith AP. Pulmonary infection with Mycobacterium kansasii in Wales, 1970-9: review of treatment and response. Thorax 1983; 38: 271-274.

8 Pang SC. Mycobacterium kansasii infections in Western Australia (1962-1987). Respir Med 1991; 85: 213-218.

9 Management of opportunistic mycobacterial infections: Joint Tuberculosis Committee Guidelines 1999. Subcommittee of the Joint Tuberculosis Committee of the British Thoracic Society. Thorax 2000; 55: 210-218.
10 Ahn CH, Lowell JR, Ahn SS, Ahn SI, Hurst GA. Shortcourse chemotherapy for pulmonary disease caused by Mycobacterium kansasii. Am Rev Respir Dis 1983; 128: 10481050 .

11 Schraufnagel DE, Stoner R, Whiting E, Snukst-Torbeck G, Werhane MJ. Testing for isoniazid. An evaluation of the Arkansas method. Chest 1990; 98: 314-316.

12 Gullans CR Sr. Digestion-decontamination procedures. In: Isenberg HD, ed. Clinical Microbiology Procedures Handbook. Washington, American Society for Microbiology, 1992: pp. 3.4.1-3.4.14.

13 Nolte FS, Metchock B. Mycobacterium. In: Murray PR, Baron EJ, Pfaller MA, Tenover FC, Yolken RH, eds. Manual of Clinical Microbiology. 6th Edn. Washington, American Society for Microbiology, 1995; pp. 400-437.

14 National Committee for Clinical Laboratory Standards. Susceptibility Testing of Mycobacteria, Nocardiae, and Other Aerobic Actinomycetes; Approved Standard. Document M24-A. Wayne, Clinical and Laboratory Standards Institute, 2003.

15 Inderlied CB, Nash KA. Antimycobacterial agents: in vitro susceptibility testing, spectra of activity, mechanisms of action and resistance, and assays for activity in biologic fluids. In: Lorian V, ed. Antibiotics in Laboratory Medicine. 4th Edn. Baltimore, Williams \& Wilkins Co., 1996; pp. 127-175.

16 Inderlied CB, Pfyffer GE. Susceptibility test methods: Mycobacteria. In: Murray PR, Baron EJ, Jorgensen JH, Pfaller MA, Yolken RH, eds. Manual of Clinical Microbiology. 8th Edn. Washington, American Society for Microbiology, 2003; pp. 1149-1177.

17 Kaustová J, Chmelík M, Ettlová D, Hudec V, Lazarová H, Richtrová S. Disease due to Mycobacterium kansasii in the Czech Republic: 1984-89. Tuber Lung Dis 1995; 76: 205-209.

18 Sauret J, Hernández-Flix S, Castro E, Hernández L, Ausina V, Coll P. Treatment of pulmonary disease caused by Mycobacterium kansasii: results of 18 versus 12 months' chemotherapy. Tuber Lung Dis 1995; 76: 104-108.

19 Mycobacterium kansasii pulmonary infection: a prospective study of the results of nine months of treatment with rifampin and ethambutol. Research Committee, British Thoracic Society. Thorax 1994; 49: 442-445.

20 Alcaide F, Calatayud L, Santín M, Martín R. Comparative in vitro activities of linezolid, telithromycin, clarithromycin, levofloxacin, moxifloxacin, and four conventional antimycobacterial drugs against Mycobacterium kansasii. Antimicrob Agents Chemother 2004; 48: 4562-4565.

21 Guna R, Muñoz C, Domínguez V, et al. In vitro activity of linezolid, clarithromycin and moxifloxacin against clinical isolates of Mycobacterium kansasii. I Antimicrob Chemother 2005; 55: 950-953.

22 Cynamon MH, Elliott SA, DeStefano MS, Yeo AE. Activity of clarithromycin alone and in combination in a murine model of Mycobacterium kansasii infection. I Antimicrob Chemother 2003; 52: 306-307.

23 Griffith DE, Brown-Elliott BA, Wallace RJ Jr. Thrice-weekly clarithromycin-containing regimen for treatment of Mycobacterium kansasii lung disease: results of a preliminary study. Clin Infect Dis 2003; 37: 1178-1182. 